\title{
Reflex responses to combined hip and knee motion in human chronic spinal cord injury
}

\author{
Ming Wu, PhD; ${ }^{*}$ Brian D. Schmit, PhD ${ }^{2}$ \\ ${ }^{1}$ Sensory Motor Performance Program, Rehabilitation Institute of Chicago, Chicago, IL, and Department of Physical Medi- \\ cine and Rehabilitation, Northwestern University Medical School, Chicago, IL; ${ }^{2}$ Department of Biomedical Engineer- \\ ing, Marquette University, Milwaukee, WI
}

\begin{abstract}
The relative contributions of hip and knee proprioceptors to the origination of extensor spasms were examined in 11 subjects with chronic spinal cord injury (SCI). Ramp and hold extension and combined hip and knee oscillation movements were imposed to the right leg while the ankle was held in a static position by a custom-designed robot. Isometric joint torques of the hip, knee, and ankle and surface electromyograms (EMGs) from seven leg muscles were recorded following controlled hip and knee extension. A stereotypical torque response consisting of hip flexion, knee extension, and ankle plantar flexion was observed following hip and knee perturbations. Further, the hip or knee joint posture modulated the spastic reflexes triggered by the extension movement of the other joint, with larger responses observed with the hip and knee extended. In addition, combined hip and knee oscillation movements were imposed to one leg with four different phasing conditions. The phasing between the hip and knee modulated the reflex activity triggered by hip and knee oscillations. The EMG patterns of the spastic reflexes were generally consistent with muscle timing during locomotion in human SCI. This knowledge may help identify rehabilitation strategies that produce functional movements in human SCI.
\end{abstract}

Key words: EMG, extensor spasms, hip and knee extension, locomotion, reflex, rehabilitation, sartorius, sensory afferents, spasticity, spinal cord injury.

\section{INTRODUCTION}

Extensor spasms differ from classically described spasticity, which is defined in the context of hyperexcitable stretch reflexes [1], although newer definitions have been proposed [2]. Extensor spasms consist of muscle activity across multiple joints, including muscles that are nonstretched or even shortened [3-5]. Despite the fact that extensor spasms are a common complication in humans with spinal cord injury (SCI), with the prevalence estimated at approximately 82 percent [6-8], the precise triggers for multijoint extensor spasms are not well understood. Clinical observations suggest that proprioceptive stimuli from the hip and knee are the most likely triggers for extensor spasms. Specifically, individuals with SCI experience extensor spasms when shifting from a sitting to a supine position (i.e., bilateral hip extension) [6,9]. In addition, manual manipulation of one leg using an imposed hip and knee extension movement acts as a relatively potent trigger

\footnotetext{
Abbreviations: $\mathrm{ADD}=$ hip adductors, $\mathrm{ANOVA}=$ analysis of variance, ASIA = American Spinal Injury Association, $\mathrm{CHKE}=$ combined hip and knee extension movement, EMG = electromyogram, HEKE = hip extension movement with knee extended, HEKF = hip extension movement with knee flexed, KEHE $=$ knee extension movement with hip extended, KEHF = knee extension movement with hip flexed, MG = medial gastrocnemius, $\mathrm{MH}=$ medial hamstrings, $\mathrm{RF}=$ rectus femoris, $\mathrm{SCI}=$ spinal cord injury, $\mathrm{SD}=$ standard deviation, $\mathrm{SOL}=$ soleus, $\mathrm{ST}=$ semitendinosus, $\mathrm{TA}=$ tibialis anterior, $\mathrm{VM}=$ vastus medialis.

*Address all correspondence to Ming Wu, PhD; Sensory Motor Performance Program, Rehabilitation Institute of Chicago, 345 East Superior Street, Room 1406, Chicago, IL 60611; 312-238-0700; fax: 312-238-2208.

Email: w-ming@Northwestern.edu

DOI:10.1682/JRRD.2009.07.0093
} 
for extensor spasms [5]. Since spasms may hinder activities of daily living, interfere with transfers and sleep, and cause pain to patients, especially those with incomplete SCI [10], an improved understanding of the neural mechanisms would be helpful in the prevention and treatment of spasms.

In controlled laboratory tests, imposed hip or knee extension movements, applied separately, trigger multijoint spastic reflexes that are consistent with clinical observations of extensor spasms in human SCI [3-4]. As a result, we postulate that the interaction of both the hip and knee proprioceptors plays a significant role in triggering extensor spasms. Hip proprioceptive stimuli have been shown to be a dominant trigger for spastic reflexes. For instance, imposed ramp and hold hip extension movement produces multijoint spastic reflexes in human SCI [3]. Furthermore, coordinated muscle activities are produced by imposed hip oscillations [11-12]. Although these previous studies highlight the contribution of hip proprioceptors to the spastic reflexes, the knee was maintained in an extended posture during the hip movements to produce the spastic responses. In a similar manner, imposed knee extension movement alone produces a comparable multijoint reflex response in human SCI [4]. These observations suggest that extensor spasms are mediated by polysynaptic pathways involving activation of organized interneuronal circuits located within the isolated spinal cord that are modulated similarly by both hip and knee proprioceptive inputs. However, the interaction between these two joint afferents in triggering spastic reflexes and the potential link between these spastic reflexes and locomotor patterns remain unclear.

The objective of this study was to characterize the relative role of the hip and knee proprioceptors in triggering extensor spasms in human SCI. We hypothesized that reflex responses triggered by hip and knee oscillation movements would depend on the relative phasing of the hip and knee. Specifically, we postulated that hip/knee phasing that is similar to nondisabled walking would produce a muscle activity pattern that resembles the pattern during gait.

\section{MATERIALS AND METHODS}

\section{Subjects}

Eleven subjects with the clinical features described in Table 1 were recruited to this study. Inclusion criteria included a history of SCI (>12 months) with associated spasticity. Participants (mean age: 40, range: 25-68)
Table 1.

Subject clinical characteristics.

\begin{tabular}{cccccl}
\hline Subject & $\begin{array}{c}\text { Level of } \\
\text { Injury }^{*}\end{array}$ & $\begin{array}{c}\text { ASIA } \\
\text { Class }\end{array}$ & $\begin{array}{c}\text { Age } \\
\text { (yr) }\end{array}$ & $\begin{array}{l}\text { Time Since } \\
\text { Injury (yr) }\end{array}$ & \multicolumn{1}{c}{ Medication } \\
\hline A & T10-11 & A & 26 & 8 & $\begin{array}{l}\text { Baclofen } \\
\text { (intrathecal pump) }\end{array}$ \\
& & & & & Baclofen (80 mg) \\
B & C5 & C & 25 & 4 & None \\
C & C7-C8 & A & 32 & 6 & Baclofen \\
D & C6 & C & 43 & 16 & (40 mg 3×/day) \\
& & & & & None \\
E & C5-C6 & C & 45 & 18 & None \\
F & T6 & A & 24 & 7 & Baclofen (100 mg) \\
G & T1 & D & 68 & 5 & None \\
H & T8 & A & 44 & 28 & None \\
I & C5-C6 & C & 41 & 24 & None \\
J & C5 & B & 44 & 27 & None \\
K & C6-C7 & D & 45 & 22 &
\end{tabular}

${ }^{*}$ Neurological injury levels: $\mathrm{C}=$ cervical, $\mathrm{T}=$ thoracic.

ASIA = American Spinal Injury Association.

included both complete (American Spinal Injury Association [ASIA] classification A) and incomplete (ASIA B, C, and D) individuals with SCI. At the time of the study, 4 of the 11 subjects were prescribed antispastic medications (baclofen) to reduce the intensity and frequency of spastic reflexes. Exclusion criteria included multiple central nervous system lesion sites, urinary tract infection, other secondary infections, heterotopic ossification, respiratory insufficiency, significant osteoporosis, or inability to give informed consent.

\section{Test Apparatus}

The experimental apparatus for this study was an instrumented hip/knee actuator modified from a device used in a previous study [11]. Briefly, the apparatus (Figure 1) was constructed to measure the multijoint torque response to precisely controlled hip and knee movement with the subject supine on a therapy table. The hip and knee joints of the robotic system were rotated by two servomotors through a planetary gearbox and a ball-screw mechanism, respectively, while the ankle joint was secured in a static position. The system was programmed to shut down instantly if it was outside specific preset ranges. Safety screws were used as mechanical stops to restrict the motion ranges of the actuators. The foot was placed in a footplate with a clamp placed on the dorsum of the foot and a strap to secure the heel. The hip-knee and knee-ankle links were adjusted to fit individual leg size. The hip portion of the leg brace was affixed to a servomotor drive system (MT 704A1-R1C1, Kollmorgen Electro-Optical; Northampton, Massachusetts). A newly constructed knee actuator, which included a 


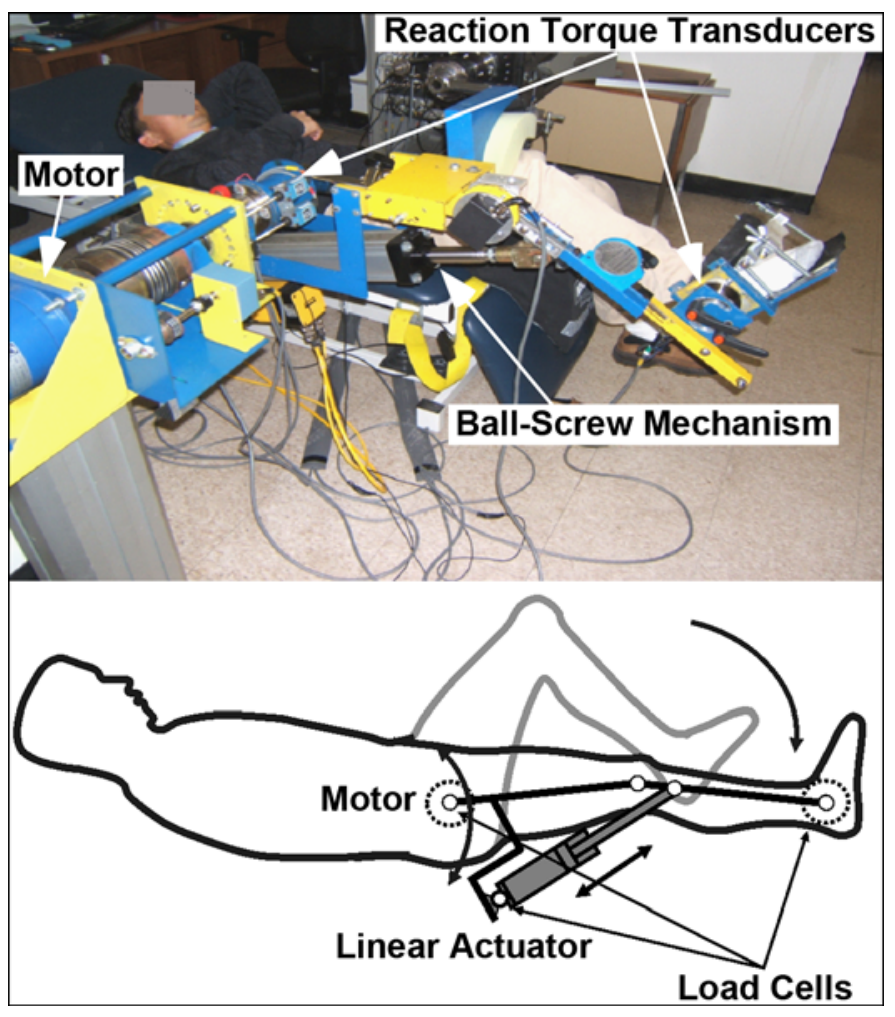

Figure 1.

Servomotor systems with leg brace attachment. Servomotor drive system actuates leg from hip and knee joints, and ankle joint is held statically by leg brace. Hip joint is driven directly by rotation motor, while knee joint is driven by motor through ball-screw mechanism and linkage. Reaction torque transducers at hip and ankle measure joint torque response to imposed movement. Reaction force transducer is attached at bottom of ball-screw mechanism to measure force from which knee joint torque is calculated.

servomotor (BE343JR-KMSN, Parker Hannifin Corporation; Rohnert Park, California), a ball-screw mechanism, and a four-bar linkage mechanism, was affixed to the kneeankle brace to impose a controlled motion to the knee. Hip and ankle torques were measured with use of hollowflanged transducers (S. Himmelstein and Company; Hoffman Estates, Illinois), while the knee torque was calculated from the force recorded by a tension and compression load cell (Honeywell Sensotec; Columbus, Ohio) mounted at the bottom of the motor. Hip and knee joint positions were measured with use of potentiometers coupled to the servomotor and rotation shaft of the linkage mechanism, respectively.

Surface electromyograms (EMGs) were recorded from the tibialis anterior (TA), soleus (SOL), medial gastrocnemius (MG), vastus medialis (VM), rectus femoris (RF), medial hamstrings (MH), and hip adductors (ADD) of the right leg in all subjects. Ag-AgCl (silver/silver chloride)
$2.5 \mathrm{~cm}$ square pregelled electrodes (Vermed Inc; Bellows Falls, Vermont) were applied with $2.5 \mathrm{~cm}$ center-to-center spacing over the respective muscle bellies on lightly abraded skin. Active preamplifiers with shielded leads were attached to the electrodes and connected to an Octopus AMT-8 EMG unit (Bortec Biomedical Ltd; Calgary, Canada). All channels were amplified (total gain $=500$ ), low-pass filtered $(450 \mathrm{~Hz})$, and sampled $(1,000 \mathrm{~Hz})$ with use of a data acquisition card (National Instruments Corporation; Austin, Texas) on a personal computer. Custom LabVIEW software (National Instruments Corporation) acquired the data and output the velocity command signals to the servomotor systems.

\section{Imposed Hip And Knee Movement}

Hip and knee movements were imposed to the right leg of all 11 subjects. Each subject was transferred to a trisection therapy table and placed in a supine position. The center of rotation of the right hip joint was aligned with the axis of rotation of the servomotor system, the knee joint was aligned with one rotation axis of a fourbar linkage mechanism, and the brace was adjusted to align the ankle with the appropriate torque transducer. Alignment of the hip and knee joints was confirmed by an absence of leg translation during manually imposed flexion and extension of the joint, assessed visually. The pelvis was secured to the table with a strap across the iliac crest to inhibit pelvic rotation. The leg was placed in the brace with the ankle at $10^{\circ}$ to $30^{\circ}$ plantar flexion and was held in the same position for the duration of the test. The contralateral limb was supported in a slightly flexed $\left(10^{\circ}\right.$ to $\left.30^{\circ}\right)$ position at the hip, with the knee at approximately $90^{\circ}$ and the ankle allowed to rest in a position determined by gravity (slightly plantar flexed).

Controlled hip movements were imposed by the Kollmorgen servomotor system. The start position was set at $30^{\circ}$ to $40^{\circ}$ of hip flexion and the end position at between $10^{\circ}$ of hip flexion and $10^{\circ}$ of hip extension, a position that corresponded approximately to maximum hip extension, depending on each subject's range of motion. Similarly, the controlled knee movements were imposed with use of the ball-screw mechanism and Kollmorgen servomotor system. The start position was set at $70^{\circ}$ to $75^{\circ}$ of knee flexion and the end position at $5^{\circ}$ to $40^{\circ}$ of knee flexion, a position that corresponded approximately to full knee extension for each subject. Because range of motion differed among subjects, a slightly different hip and knee range of movement was used for each subject (Table 2). Figure 1 shows the motion of the hip and knee relative to the sagittal coordinate 
Table 2.

Subject test parameters indicating starting position and range of motion of hip and knee joints and static ankle angle during movement.

\begin{tabular}{cccccc}
\hline Subject & $\begin{array}{c}\text { Ankle Plantar } \\
\text { Flexion Angle } \\
\left(\mathbf{(}^{\circ}\right.\end{array}$ & $\begin{array}{c}\text { Knee Flexion } \\
\text { Angle }\left({ }^{\circ}\right)\end{array}$ & $\begin{array}{c}\text { Knee } \\
\text { Range }\left({ }^{\circ}\right)\end{array}$ & $\begin{array}{c}\text { Hip Flexion } \\
\text { Angle }\left({ }^{\circ}\right)\end{array}$ & $\begin{array}{c}\text { Hip } \\
\text { Range } \\
\left({ }^{\circ}\right)\end{array}$ \\
\hline A & 20 & 70 & 52 & 40 & 50 \\
B & 20 & 70 & 65 & 40 & 50 \\
C & 15 & 75 & 62 & 40 & 40 \\
D & 20 & 75 & 50 & 30 & 40 \\
E & 25 & 75 & 61 & 30 & 30 \\
F & 20 & 75 & 61 & 30 & 30 \\
G & 25 & 75 & 62 & 40 & 50 \\
H & 15 & 75 & 60 & 40 & 30 \\
I & 10 & 75 & 35 & 40 & 40 \\
J & 30 & 75 & 48 & 30 & 40 \\
K & 15 & 70 & 48 & 30 & 20 \\
\hline \hline
\end{tabular}

system. A ramp and hold movement was imposed to the hip in which the motor rotated the hip at a constant velocity of $60 \%$, from the hip flexed to the hip extended and back (a total range of $20^{\circ}$ to $50^{\circ}$, depending on the subject), with a 10 -second hold in extension. A similar perturbation was imposed to the knee, with its velocity set at about $67^{\circ} / \mathrm{s}$ (a total range of $35^{\circ}$ to $65^{\circ}$, depending on the subject). This perturbation, consisting of controlled hip and/or knee extension movement, produces a stereotypical reflex response that resembles an extensor spasm [3-4].

Five different types of perturbation were applied to the hip and knee joints: combined hip and knee extension movement (CHKE), hip extension movement with knee flexed (HEKF) or extended (HEKE), and knee extension movement with hip flexed (KEHF) or extended (KEHE). Each set of conditions was repeated three times, with the mean of the three measurements in each condition used for statistical analyses. The five conditions were applied in random order for each set of trials.

Following the tests of ramp and hold movements, combined hip and knee oscillation movements were imposed to the leg for 10 cycles with four different phasing conditions: (1) phase 0: the hip and knee were moved in the same phase, i.e., the hip and knee reached flexed and extended positions simultaneously; (2) phase 0.50: the hip and knee were moved $180^{\circ}$ out of phase, i.e., the hip reached a flexed position while the knee reached an extended position and vice versa; (3) phase 0.25 : the hip was moved $90^{\circ}$ ( $1 / 4$ of the cycle) earlier than the knee; and (4) phase 0.75: the hip was moved $270^{\circ}$ (3/4 of the total cycle) earlier than the knee. A time interval of about 3 minutes was allowed between trials. Each set of condi- tions was repeated three times, with the four phasing conditions randomized for each set of trials.

\section{Analysis}

\section{Ramp Stretch Movements}

The reflex joint torque data were obtained for each movement trial during the 10 -second hold period with the hip (or knee) extended and the other joint, i.e., knee (or hip) flexed or extended. The torque measurements recorded immediately at the end of the movements were excluded from the analysis because of large inertial artifacts. During the hold periods, the gravitational/passive torque offset was removed by subtraction of a torque measurement in the end position, when there was no muscle activity (i.e., at the end of the hold period). The torque signals were then low-pass filtered at $5 \mathrm{~Hz}$ with use of a fourth-order Butterworth filter (butter/filtfilt; MATLAB Command, The MathWorks; Nattick, Massachusetts).

The peak torque of the hip, knee, and ankle during the hold period were the primary measurements used to compare the reflex response across conditions. The peak torques of the hip, knee, and ankle joints were identified for each movement trial in each condition. An outlier analysis was conducted on the data, and two subjects were excluded from further analysis. One subject produced torque responses $>3$ standard deviation (SD) above the mean, and the other subject produced no response. The peak torques of the hip, knee, and ankle were compared across five movement conditions with use of a one-way repeated measures analysis of variance (ANOVA) $(n=9)$. Significance was tested at $\alpha=0.05$.

The EMG signals were also used to compare the spastic reflex across different conditions. The surface EMG signals were rectified and enveloped with use of an $8 \mathrm{~Hz}$, lowpass, fourth-order Butterworth filter (butter/filtfilt; MATLAB Command, The MathWorks). The area of the rectified and smoothed EMG signals was calculated during the hold period (from the end of the movement, for 5 seconds) for the five different test conditions. A one-way ANOVA was used to statistically compare the EMG activities across the five different conditions ( $\alpha=0.05)$.

\section{Sinusoidal Movements}

For the sinusoidal movement tests, smoothed rectified EMG signals were analyzed to identify the effect of the modulation of the phasing between the hip and knee on the muscle activity pattern. The surface EMGs were 
rectified and enveloped with use of an $8 \mathrm{~Hz}$, low-pass, fourth-order Butterworth filter (butter/filtfilt; MATLAB Command, The MathWorks). The area of the rectified and smoothed EMG signals was calculated for the middle six cycles across the four different phasing conditions, i.e., phases 0, 0.25, 0.50, and 0.75. (Note that the EMG signals from the very beginning and the ending of the movement were excluded because of the transitions at those points.) The EMG areas were averaged across six cycles for each phase condition and then normalized to the average of the four phase conditions. A one-way repeated measures ANOVA was used to statistically compare the EMG activities across four different phasing conditions, with the significance set at $\alpha=0.05$.

Muscle timing patterns during each sinusoidal cycle were examined with use of circular statistics according to methods outlined by Batschelet [13] and previously applied to imposed hip movements [11-12]. The EMG phase analysis was pursued only if the EMG data demonstrated sufficient muscle activity. The threshold values were determined with use of the area of rectified and smoothed EMG data; the data were excluded if the area of each cycle was less than 15 percent of the averaged area across all six cycles and three trials. Phase analysis consisted of the use of rectified and smoothed EMG signals that were normalized from $0^{\circ}$ to $360^{\circ}$, where $180^{\circ}$ represents full hip and knee extension and $0^{\circ}$ represents full hip and knee flexion. For each movement cycle, the rectangular coordinates $(x, y)$ of the resultant vector were calculated, respectively, as shown in Equations (1) and (2):

$$
\bar{x}=\frac{1}{n}\left[\cos \left(\varphi_{1}\right)+\cos \left(\varphi_{2}\right)+\ldots+\cos \left(\varphi_{n}\right)\right]
$$

and

$$
\bar{y}=\frac{1}{n}\left[\sin \left(\varphi_{1}\right)+\sin \left(\varphi_{2}\right)+\ldots+\sin \left(\varphi_{n}\right)\right],
$$

where $\varphi_{1} \sim \varphi_{n}$ were the observed angles of the corresponding unit vectors.

For these rectangular coordinates, the phase angle for each cycle was then calculated with use of Equation (3). To characterize the phasing across cycles, we normalized the vector length $r$ to a unit vector and converted the polar coordinates to rectangular coordinates to find the mean $x$ and $y$ coordinates. After normalization, the mean phase angles $\bar{\varphi}$ and vector lengths $\bar{r}$ were then calculated
(Equations (3) and (4), respectively) across all six cycles and three trials for each subject.

$$
\bar{\varphi}=\arctan \left(\begin{array}{l}
\bar{y} \\
\bar{x}
\end{array}\right)
$$

and

$$
r=\sqrt{\bar{x}^{2}+\bar{y}^{2}}
$$

Only the middle six cycles of each trial were used for analysis, and the first and last two cycles were discarded because of the unsynchronized onset of hip and knee movement for different phase-shift conditions. The mean phase angles and vector lengths were used to detect significant phasic muscle activity during the imposed hip and knee oscillation.

To determine whether significant phasing in EMG existed, we performed Raleigh's test for one-sidedness ( $\alpha=$ $0.05)$ [13]. All data sets that showed a significant trend were plotted in polar coordinates. To identify the possible contribution of the stretch reflex to the muscle activities triggered by hip and knee motion, we estimated the time intervals in which muscles were being stretched. The length changes of the biarticular hip/knee muscles were obtained with the model described by Frigo and Pedotti [14]. The model was applied to the RF and to the semitendinosus (ST), the latter chosen to represent the MH. We compared times of muscle lengthening with timing of muscle activity measured experimentally to aid interpretation of the results by identifying the possible contribution of stretch reflexes to the overall observed responses.

\section{RESULTS}

\section{Ramp and Hold Movement Responses}

A typical reflex response, from subject I, is shown in Figure 2 for two perturbation conditions: CHKE and HEKF. The response to the imposed hip and knee extension movement typically consisted of hip flexion, knee extension, and ankle plantar flexion during the hold period, with peak responses at about 1.7 seconds following the end of the movement. In contrast, only modest torque responses at the knee and ankle were observed for the HEKF condition. 
(a)

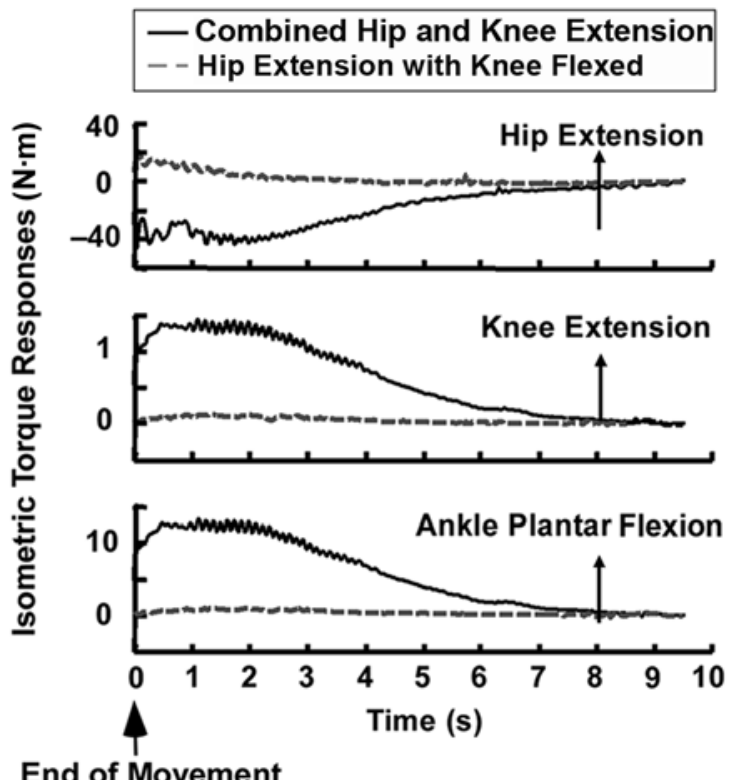

(b)
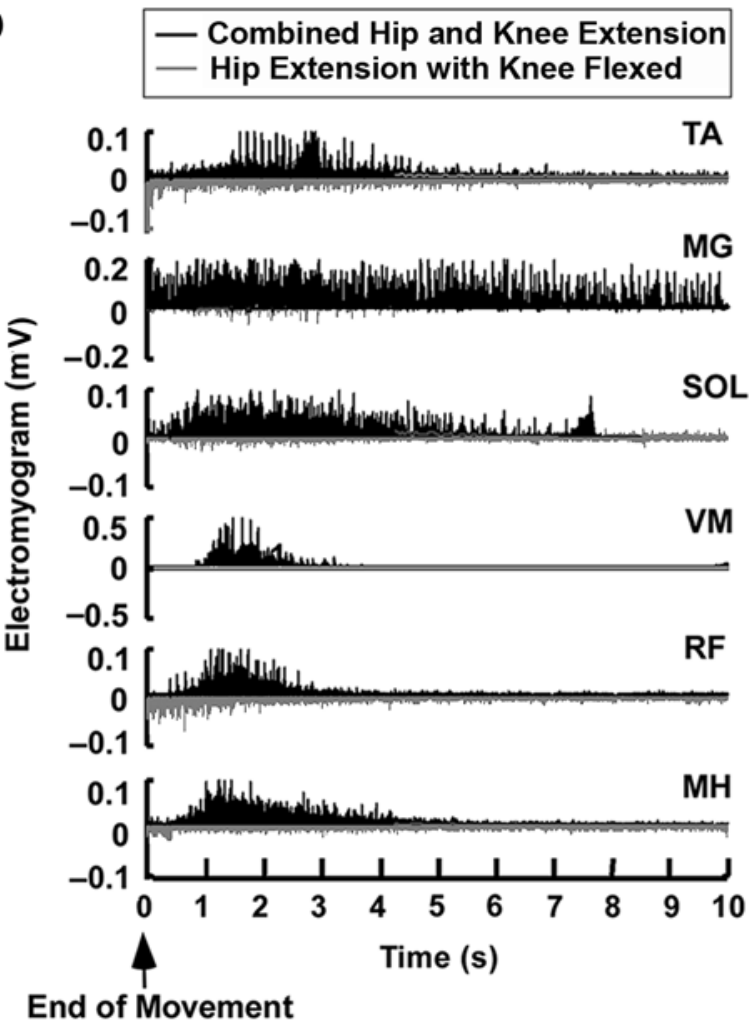

Figure 2.

(a) Example torque data for subject I, elicited by combined hip and knee extension movement and hip extension movement with knee flexed. Positive torque is defined as extension and negative torque as flexion for the hip (upper trace) and knee (middle trace). Positive torque is defined as plantar flexion and negative torque as dorsiflexion for ankle (lower trace). Following end of movement, clear pattern of isometric torque response with hip flexion, knee extension, and ankle plantar flexion was observed for condition of combined hip and knee extension movement. Modest torque responses for knee and ankle were observed for condition of hip extension movement with knee flexed. (b) Typical electromyography (EMG) responses to imposed hip and knee movement for subject I. EMG activities of tibialis anterior (TA), medial gastrocnemius (MG), soleus (SOL), vastus medialis (VM), rectus femoris (RF), and medial hamstrings (MH) were elicited with combined hip and knee movement into extension. Especially, prolonged muscle activities were observed for ankle plantar flexors (MG and SOL) during hold period, even though movement was induced only at hip and knee joints. In addition, cocontraction of knee extensors and flexors was commonly observed for this condition. In contrast, for condition of hip extension with knee flexed, only modest EMG muscle activities were observed for most leg muscles.

EMG data were consistent with the recorded torque responses, with similar patterns of activity across two conditions but larger magnitude of EMG signals for the CHKE condition. A typical surface EMG response, from subject I, to imposed hip and knee movement and hip movement with knee flexed is shown in Figure 2(b). During the hold period, the muscle activities of the knee and ankle extensors, represented by the VM, MG, and SOL, were activated by the CHKE or HEKF conditions, although the muscle activities were much smaller for the HEKF condition. The RF (a hip flexor/knee extensor) was also activated following the end of the movement, coinciding with the measured hip flexion and knee extension torques, with larger responses for the CHKE condition. The knee flexors, represented by $\mathrm{MH}$, and the ankle dorsiflexors, represented by TA, were also activated following the movement, again with larger responses for the CHKE condition.

Statistically, the knee joint position had a significant influence on the spastic reflexes triggered by hip extension movement, with larger responses occurring when the knee was in an extended position. The mean peak torque values of hip flexion, knee extension, and ankle plantar flexion across 9 of 11 subjects for the five different conditions of hip and knee movement are shown in Figure 3 (as mentioned, one subject was excluded from the analysis because of an absence of spasms and one subject was 
(a)

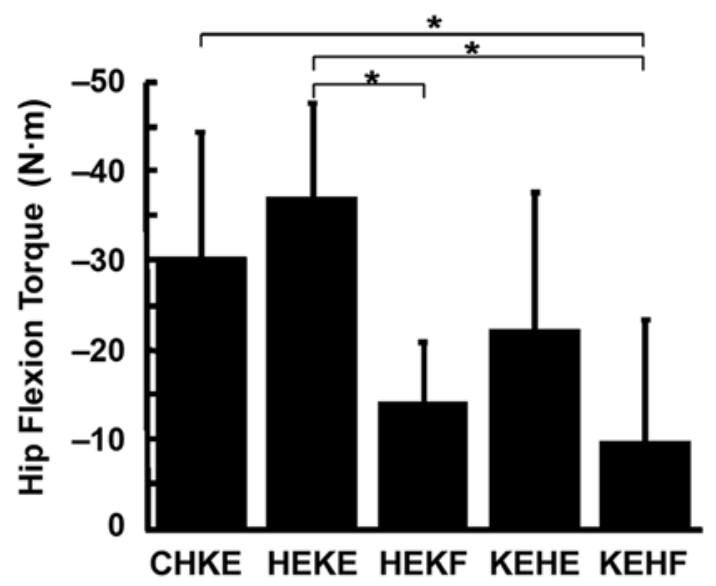

(b)

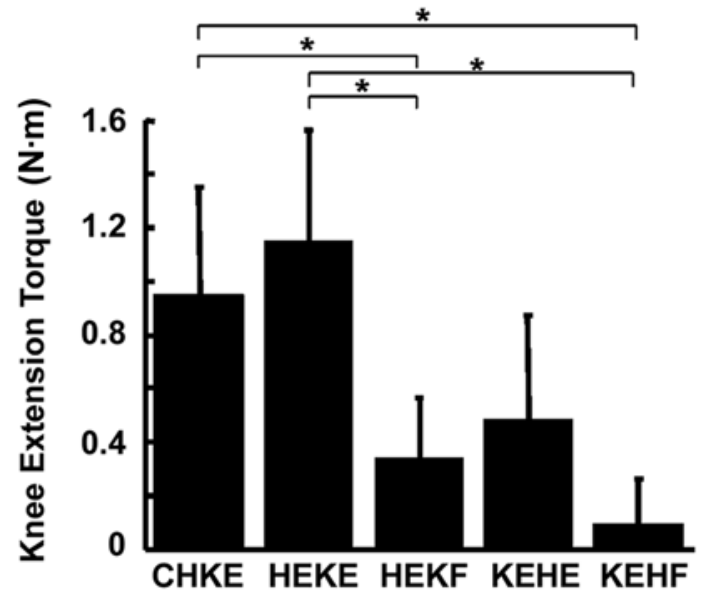

(c)

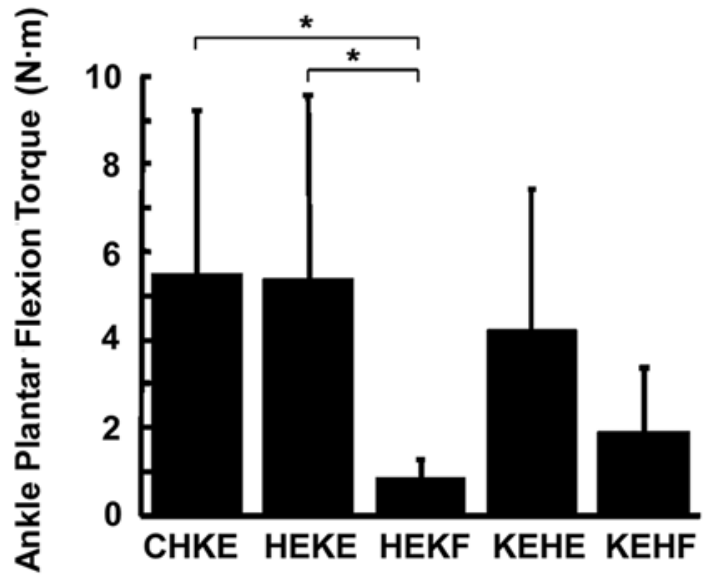

Figure 3.

Peak torque responses at three joints for nine subjects during five different conditions: combined hip and knee extension (CHKE), hip extension movement with knee extended (HEKE), hip extension movement with knee flexed (HEKF), knee extension movement with hip extended (KEHE), and knee extension movement with hip flexed (KEHF). Data represent mean \pm standard deviation for nine subjects, three trials per subject. Positive direction of torques was (a) hip extension, (b) knee extension, and (c) ankle plantar flexion. ${ }^{*}$ Significant difference $(p<0.05)$. excluded because the datum was classified as an outlier, defined as a magnitude $>3$ SD above the population mean). A one-way ANOVA (different conditions of hip and knee movement) indicated that the reflex responses to hip extension movement were significantly affected by the knee joint position $(p<0.05)$ (Figure 3). Post hoc pairs comparisons indicated that the peak torque of hip flexion was significantly greater for HEKE than for HEKF and KEHF (Tukey test, $p<0.05$ ) (Figure 3(a)). The peak hip flexion torque responses were also significantly greater for CHKE than for KEHF (Tukey test, $p<0.05$ ). No significant difference was found in peak hip flexion torque between the KEHE condition and the conditions with one joint in a flexed posture (KEHF or HEKF; $p=0.66$ and $p=$ 0.24 , respectively). The peak knee extension torque was also significantly greater with HEKE and CHKE than when one joint was in a flexed posture (HEKF or KEHF; Tukey test, $p<0.05$ ) (Figure 3(b)), despite the potential mechanical advantage associated with the more flexed knee posture [15]. The ankle plantar flexion torque was significantly larger for perturbations with biarticular extension (CHKE and HEKE) than HEKF (Tukey test, $p<$ 0.05). No significant difference was found between CHKE and KEHF or HEKE and KEHF ( $p=0.10$ and $p=0.11$, respectively) for the ankle plantar flexion torque. In summary, the torque responses were generally larger when the leg posture at the end of the imposed movement consisted of both hip and knee extension, although the magnitude of the torque responses were relatively smaller following the knee extension movement than the hip extension movement.

The results of the statistical analyses of the EMG data were similar to the torque results. A one-way ANOVA (different hip and knee movement conditions) of the integrated EMG signals of the MG, SOL, RF, VM, and ADD in 10 of the 11 subjects indicated that muscle activations were significantly affected by hip and knee joint positions ( $p<0.05$ ), with larger responses obtained when the end position included both hip and knee extension (CHKE, HEKE, and KEHE) versus when one joint was flexed (HEKF and KEHF) (one subject was excluded from analysis because of an absence of spasms). No significant difference was observed for the TA and MH across different hip and knee movement conditions ( $p=0.17$ and $p=0.07$, respectively). The magnitudes of the differences are summarized in Figure 4. 


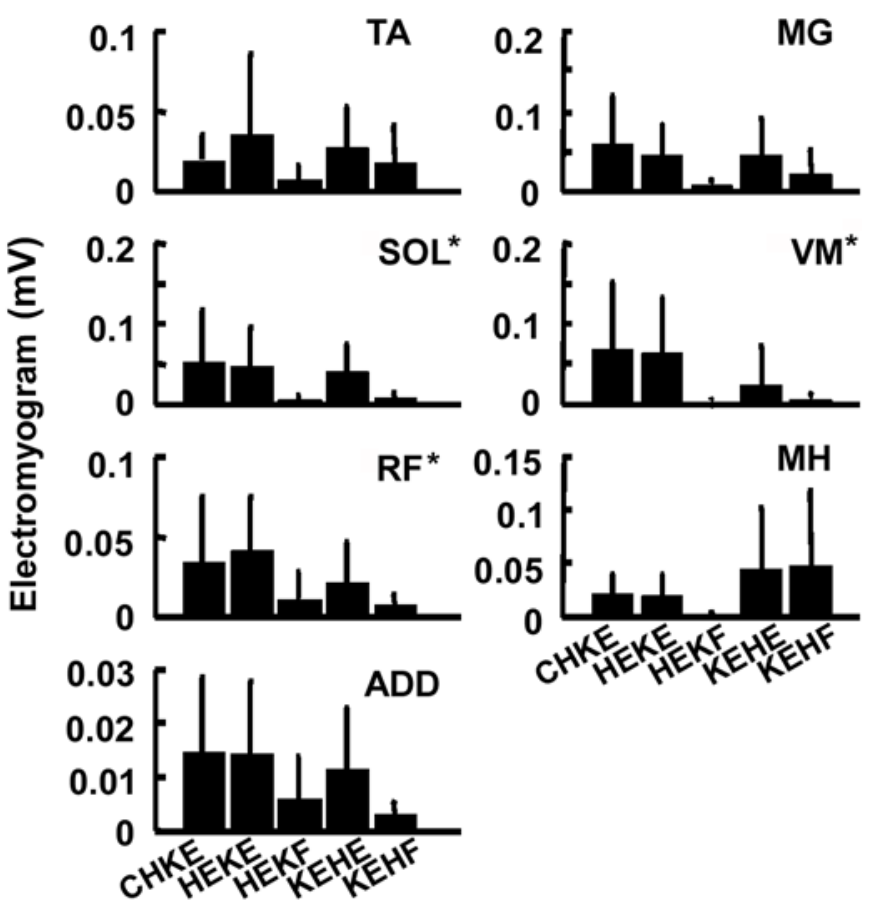

Figure 4.

Integrated electromyography activity of seven muscles: tibialis anterior (TA), medial gastrocnemius (MG), soleus (SOL), vastus medialis (VM), rectus femoris (RF), medial hamstrings (MH), and adductors (ADD) for 10 subjects. Five perturbation conditions are shown: combined hip and knee extension (CHKE), hip extension movement with knee extended (HEKE), hip extension movement with knee flexed (HEKF), knee extension movement with hip extended (KEHE), and knee extension movement with hip flexed (KEHF). Data represent mean \pm standard deviation for 10 subjects during hold period of hip or knee extension. *Significant difference $(p<0.05)$.

\section{EMG During Sinusoidal Oscillations}

The EMG responses to imposed hip and knee sinusoidal oscillations demonstrated cyclical alternating activity synchronized with the imposed movement. A typical surface EMG response to imposed hip and knee sinusoidal oscillation movement (six cycles) is shown in Figure 5 for subject $F$, with the phasing at 0.75 . A phase of 0.75 is close to the phasing between the hip and knee during nondisabled walking [16]. The alternating muscle activities of the $\mathrm{RF}, \mathrm{MH}$, and SOL are shown for this subject during imposed hip and knee sinusoidal movements.

Statistically, the magnitude of muscle activities elicited by imposed hip and knee movement depended on the phasing between the hip and knee joints. Nine out of the eleven subjects participated in the oscillation experimental paradigm. Data from eight of nine subjects were analyzed, with

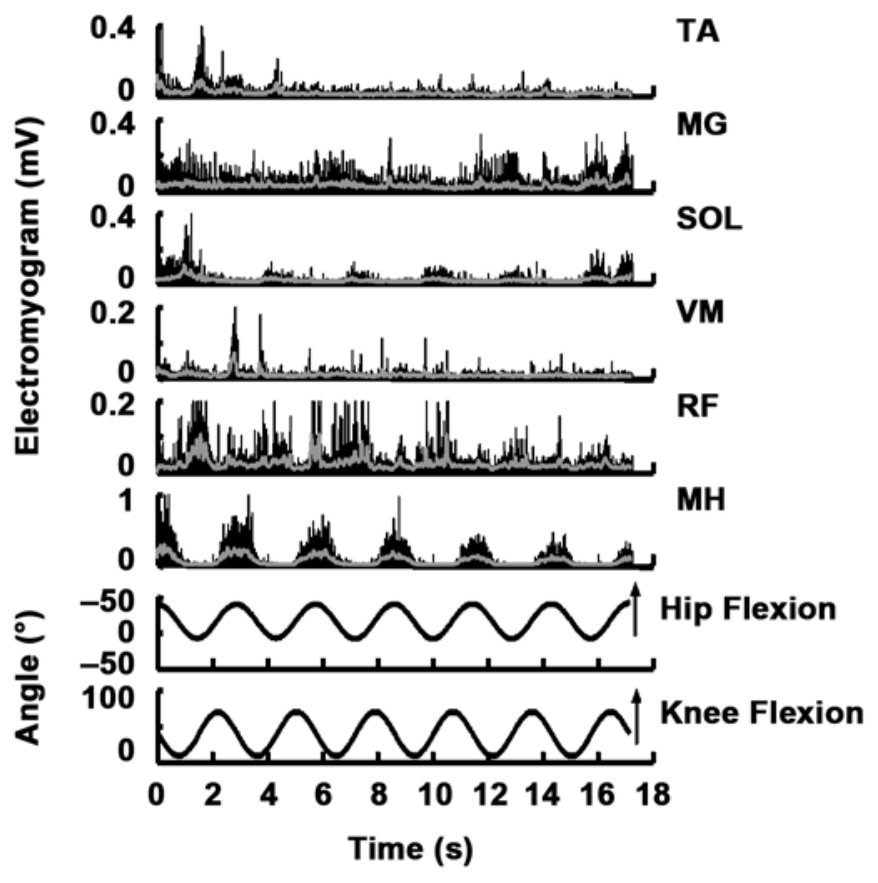

Figure 5.

Typical electromyography (EMG) responses and associated hip and knee motion profile for six cycles of trial with phasing at 0.75 (hip joint moves $3 / 4$ of a cycle $\left[270^{\circ}\right]$ earlier than knee) in subject F. Medial hamstrings $(\mathrm{MH})$ activity is in phase with imposed hip flexion movements. Soleus (SOL) and rectus femoris (RF) show activity during imposed knee flexion movements. $\mathrm{MG}=$ medial gastrocnemius, $\mathrm{TA}=$ tibialis anterior, $\mathrm{VM}=$ vastus medialis. Black and gray traces indicate rectified and smoothed EMG signals (low-pass with cutoff frequency at $8 \mathrm{~Hz}$ ), respectively.

the data from one subject excluded because of an absence of spasms. A one-way ANOVA indicated that phasing had a significant impact on the muscle activities for the TA $(n=$ $8)$, SOL ( $n=7$; one subject was excluded from analysis because the datum was classified as an outlier, defined as a magnitude $>3$ SD above the population mean), RF ( $n=7$; one subject was excluded because the datum was classified as an outlier), $\operatorname{ADD}(n=8)$, and $\mathrm{MH}(n=8)(p<0.05)$. Post hoc analyses indicated that the integrated EMG signals from RF and ADD were significantly larger for phase 0.75 than phases $0,0.25$, and 0.50 (Tukey test, $p<0.05$ ). Similarly, the integrated EMG signals from the TA were significantly smaller for phase 0 than phases 0.50 and 0.75 (Tukey test, $p<0.05$ ), with the largest value for phase 0.75 . While the integrated EMG signals from the SOL were significantly larger for phase 0 than phases 0.25 and 0.50 (Tukey test, $p<0.05$ ), the second largest value was for phase 0.75 . The EMG signals from the MH were significantly smaller for phase 0 than phases $0.25,0.50$, and 0.75 (Tukey test, $p<$ 0.05). The phasing had no significant impact on the 
integrated EMG signals for the MG $(p=0.7)$ and VM ( $p=$ $0.9)$. A summary of the integrated EMG signals from the seven muscles is shown in Figure 6.

The polar angles of the mean resultant vector for the EMG signals, which were used to determine the overall phasing of activity for each muscle, showed a phasing of the MG, SOL, VM, RF, and MH activity that depended on hip and knee positions. Figure 7 shows the angular locations of the resultant vectors, for four different phasing conditions of hip and knee movement, for trials that met Raleigh's test criteria $(p<0.05)$. A mean resultant vector phase angle of $180^{\circ}$ corresponds to muscle activity in phase with movement of the hip and knee joints into extension, while $0^{\circ}$ corresponds to muscle activity in phase with the hip and knee joints into flexion (Figure 7(a)). Each mean resultant vector (signified by an arrow in Figure 7(b)) represents the response from one subject.

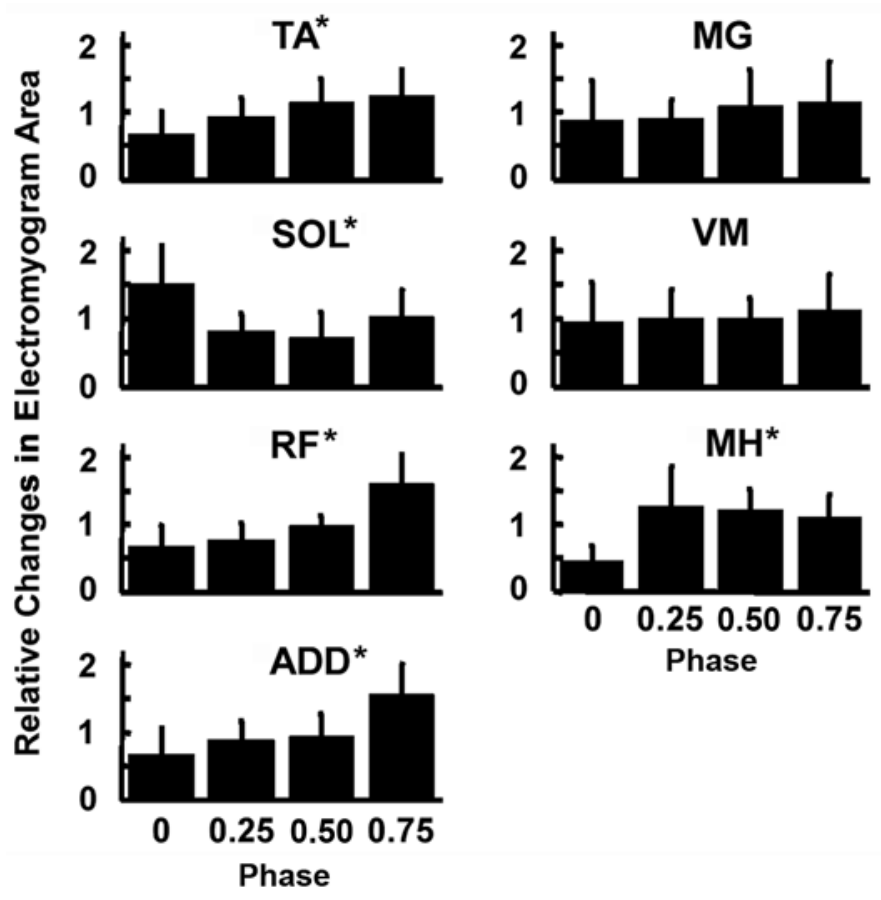

Figure 6.

Relative change in integrated electromyography (EMG) activity of seven muscles: tibialis anterior (TA), medial gastrocnemius (MG), soleus (SOL), vastus medialis (VM), rectus femoris (RF), medial hamstrings (MH), and adductors (ADD) for eight subjects. Data were averaged across six cycles and normalized to mean of integrated EMG activity across four phasing conditions: phase 0 , hip and knee joints are in same phase; phase 0.50 , hip and knee joints are out of phase; phase 0.25 , hip is $1 / 4$ cycle $\left(90^{\circ}\right)$ earlier than knee joint; phase 0.75 , hip is $1 / 4$ cycle $\left(90^{\circ}\right)$ later than knee joint. Data represent mean \pm standard deviation. ${ }^{*}$ Significant difference $(p<0.05)$. (a)
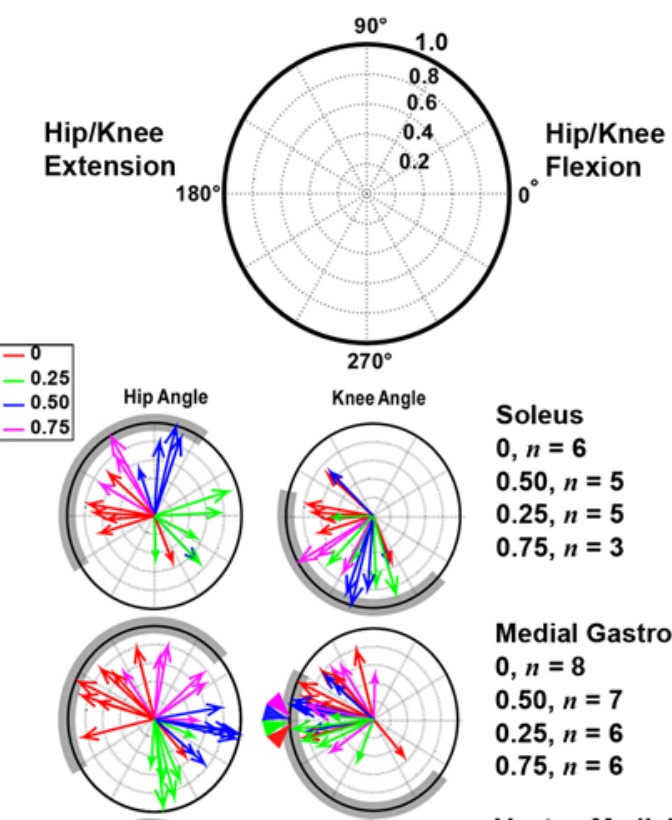

Medial Gastrocnemius

$0, n=8$

$0.50, n=7$

$0.25, n=6$

$0.75, n=6$

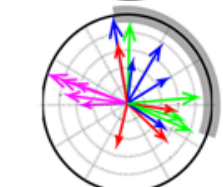

Vastus Medialis

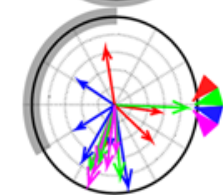

$0, n=4$

$0.50, n=5$

$0.25, n=4$

$0.75, n=5$

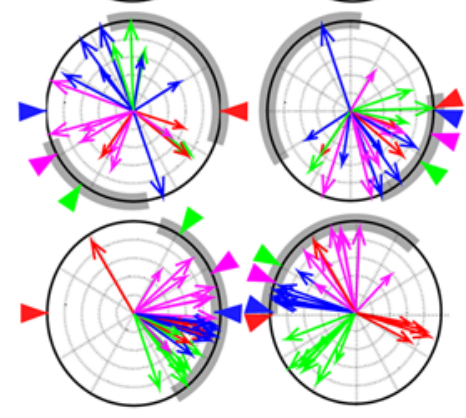

Rectus Femoris

$0, n=3$

$0.50, n=7$

$0.25, n=4$

$0.75, n=7$

Medial Hamstrings

$0, n=5$

$0.50, n=8$

$0.25, n=8$

$0.75, n=8$

Figure 7.

Phase relationship between hip and knee position and muscle activity from one leg. (a) Full hip and knee flexion corresponds to $0^{\circ}$ and full hip and knee extension corresponds to $180^{\circ}$ for each polar plot. Direction of hip and knee motion is counterclockwise on polar plots. (b) Significant muscle phasing patterns for hip and knee oscillation (Rayleigh's test, $\alpha<0.05$ ). Directions of plotted vectors (arrows) represent mean timing of muscle activity during four different phasing conditions $(0,0.25,0.50$, and 0.75$)$. Vector magnitude (radius $=1$ is maximum) represents uniformity of muscle timing during hip and knee movement. Number of subjects $(n)$ demonstrating significant phasing patterns are listed by each plot. Shaded bars on each plot indicate timing and duration of muscle activation corresponding to hip and knee trajectories during normal locomotion (Hoy MG, Zajac FE, Gordon ME. A musculoskeletal model of the human lower extremity: The effect of muscle, tendon, and moment arm on the momentangle relationship of musculotendon actuators at the hip, knee, and ankle. J Biomech. 1990;23(2):157-69. [PMID: 2312520] DOI:10.1016/00219290(90)90349-8). Positions when muscles were maximally stretched are marked with different color arrows for different phase conditions: red arrow represents phase 0 , green arrow represents phase 0.25 , blue arrow represents phase 0.50 , and pink arrow represents phase 0.75 . 
The phasing arrows show that for the majority of the trials, the SOL and MG were phased with knee extension (with the exception of one subject for the MG), while the RF and VM were phased with knee flexion (with the exception of one subject for the RF and two subjects for the VM). This phasing pattern is reflected by a tighter grouping of vectors in the plots of SOL, MG versus knee angle, and RF, VM plots versus hip angle, respectively. In the majority of the trials, the $\mathrm{MH}$ phased with hip flexion (except for one subject). No apparent phasing trend was observed for TA versus the hip and knee position (not shown in Figure 7). In addition, the phasing of $\mathrm{MH}$ versus the hip position was affected slightly by knee phasing, and MG, SOL versus knee angle was influenced by the hip phasing. These lesser influences were observed as shifts in the plots of the phasing of muscle activity of MG, SOL versus knee position, and $\mathrm{MH}$ versus hip position, depending on the phase of the opposite joint. A summary of the phasing of six muscles is shown in Figure 7(b). For comparison, the timing of muscle activation versus hip and knee position during nondisabled locomotion is represented by the gray bars in Figure 7(b) [16]. The activity in most muscles was appropriately phased with the position of the hip and knee corresponding to nondisabled walking, when the phasing of hip and knee movement was similar to the nondisabled walking pattern, i.e., phase 0.75 . However, the muscle activity of the VM for phase 0.75 was antiphased with the position of the hip and knee corresponding to nondisabled walking.

\section{DISCUSSION}

Following imposed hip and knee combination extension movements, a reflex pattern of hip flexion, knee extension, and ankle plantar flexion was observed in 10 of 11 subjects with chronic SCI. These responses were consistent with clinical descriptions of the extensor spasms that are observed following movement from a sitting to a supine position [6]. The reflex response to hip or knee extension movement depended on the position of the other joint, with greater reflex responses occurring when the other joint was extended. Furthermore, the largest muscle activity occurred when the phasing of hip and knee movement was closest to the nondisabled walking pattern. These results suggested that proprioceptive information from both the hip and knee joints contributes to a common multijoint reflex that may be, at least in part, involved in the neural pathway of locomotion in human SCI.

\section{Role of Interneuronal Excitability in SCI Spasticity}

Results from the current study emphasize that spastic reflexes in chronic human SCI include multijoint responses, suggesting the involvement of interneuronal pathways. For instance, hip or knee extension alone or simultaneous hip and knee extension triggered a multijoint response that included prominent activity in the SOL, which is not stretched or shortened by the imposed knee and hip movements. These results are consistent with previous studies indicating that subjects with chronic SCI produce multijoint reflex responses to single joint movements at other joints, such as flexor reflexes triggered by movement of the ankle [17] and knee joints [18] and extensor reflexes triggered by movement of the hip or knee [3-4]. The multijoint nature of these reflex responses supports the notion that spastic reflexes in chronic SCI can be attributed, at least in part, to an increased excitability in the coupling between specific modules within the spinal cord.

The reflex response to imposed hip and knee extension movement might involve the excitation of common interneuronal pathways originating from the proprioceptive afferents of a single biarticular hip/knee extensor. The torque pattern of ankle plantar flexion, knee extension, and hip flexion was triggered by hip or knee extension alone or simultaneous hip and knee extension. The multijoint nature of the response, which included activation of nonstretched muscles, suggests that the movement activated spinal centers that control multiple muscle groups. The position of the hip or knee joint influenced the magnitude of extensor spasms triggered by movement of the other joint. As a result, we postulate that a coupling between hip and knee afferents exists when triggering extensor spasms, possibly involving sensory signals from muscles that cross both hip and knee joints. The sartorius, a combined hip and knee flexor, may play an important role as the sensor in triggering the response.

Another potential trigger for the extensor spasms observed in the current study is a reflex chain in which imposed hip and knee extension produce stretch reflex activation of the hip and knee flexors during the movement [19-20], which, in turn, produces a flexor reflex response [21-22] that then activates the neural circuitry associated with extensor spasms. However, extensor spasms are considerably reduced when the hip or knee is flexed, suggesting stretch-related afferents from hip or knee flexors may not readily account for the observed multijoint reflexes. 


\section{Spasticity Management in Human SCI}

The findings from this study emphasize the pervasive nature of extensor spasm activity in human SCI and have potential implications for the management and clinical assessment of spastic motor behaviors. Extensor spasms are manifested more frequently than other types of spastic reflexes [6] and are often considered more disabling than other spastic motor behaviors. For instance, extensor spasms have been known to force a patient out of a wheelchair and to interfere with transfers $[7,23]$. Note that the responses observed in the current study include hip flexion torque and thus resemble "extensor spasms" observed clinically when patients with SCI move from a sitting to a supine posture. Other types of extensor spasms are observed clinically that can involve strong hip extension. The mechanism for these extensor spasms likely differs from the responses observed in the current study, likely involving a different sensory trigger.

While extensor spasms appeared to dominate the response to ramp and hold perturbations, stretch reflexes likely contributed to the muscle activity pattern triggered by hip and knee oscillation. The MG activity was generally timed with stretch, reflected as timing consistent with knee extension (Figure 7(b)). In addition, integrated muscle activities of the RF and $\mathrm{MH}$ were smaller for phase 0 and larger for phases 0.50 and 0.75 (Figure 6). This difference is consistent with estimates of muscle stretch, where the overall muscle length change was smaller for phase 0 and larger for phases 0.50 and 0.75 (Figure 8(a) and (b)). The phase of the peak muscle activity was also consistent with the results from muscle length estimates for the $\mathrm{MH}$ and, to a lesser extent, RF. For example, phasing of the MH occurred mostly at peak hip flexion, where the ST was stretched the most at phases 0.50 and 0.75 (Figure 8). Less consistency was seen between the phasing arrows and the estimated muscle length change of the RF, suggesting that reflex pathways other than homonymous stretch reflexes are likely involved in the muscle activity pattern triggered by hip and knee oscillation.

The existence of multijoint reflexes with resemblance to extensor spasms was also evident in hip and knee oscillation perturbations. SOL muscle activity was observed despite the fact that the ankle was held in a static position. Further, VM activity was not phased with peak muscle stretch. These observations, in addition to the previously described RF muscle activity timing,

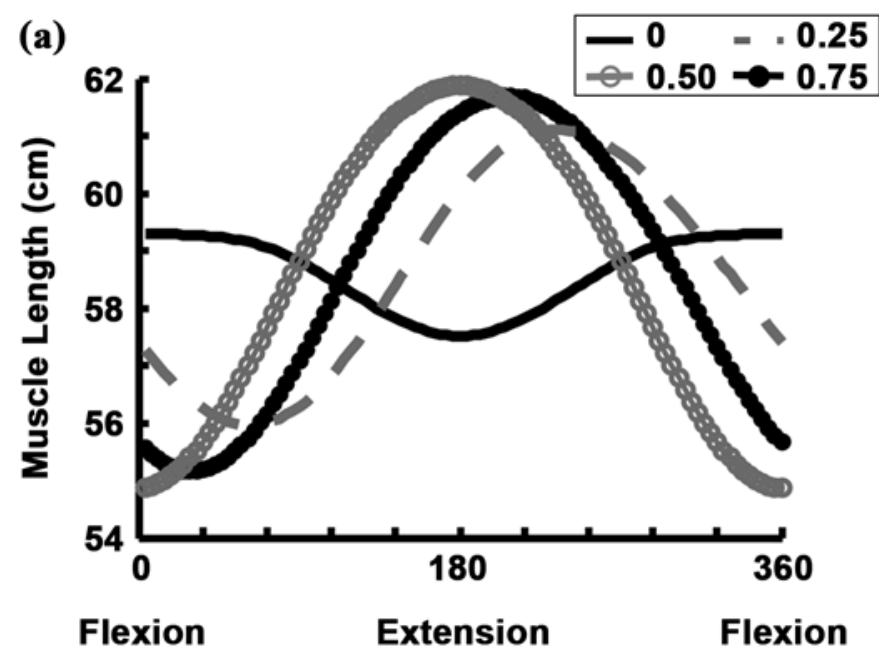

Hip Cycle Position ( $\left.{ }^{\circ}\right)$

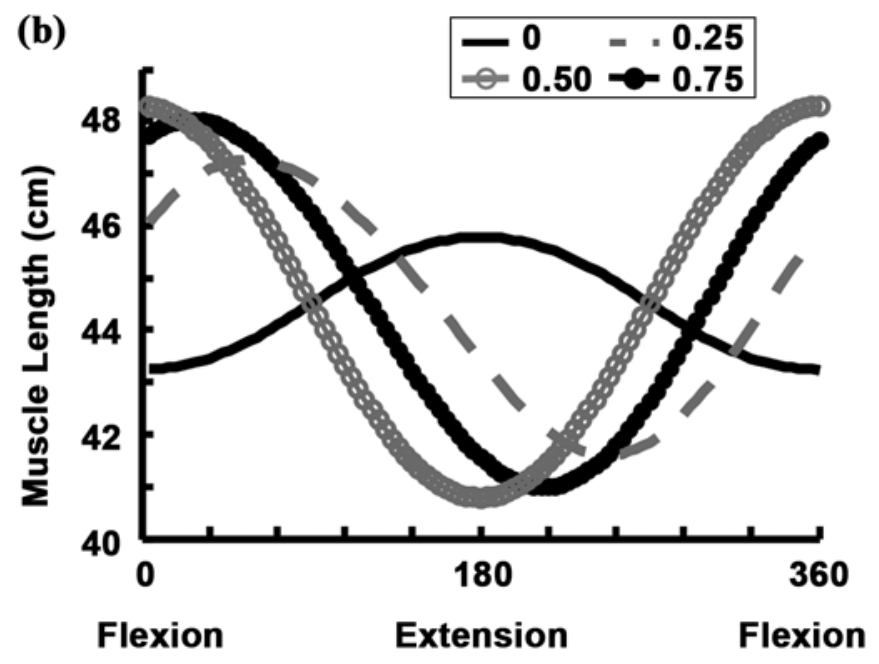

Hip Cycle Position ( $\left.{ }^{\circ}\right)$

Figure 8.

Muscle length estimation for (a) rectus femoris and (b) semitendinosus. Four different phase conditions (0, 0.25, 0.50, and 0.75) are shown. Lengths of muscles were estimated from averaged hip and knee joints angle across 11 subjects and model described by Frigo and Pedotti (Frigo C, Pedotti A. Determination of muscle length during locomotion. In: Asmussen E, Jørjensen K, editors. Biomechanics VI. Baltimore (MD): University Park Press; 1978. p. 355-60).

implicate the existence of a more complex reflex response. In total, hip and knee oscillations appear to invoke both stretch reflex and extensor spasm spinal networks, creating a complex, vigorous response to imposed hip and knee perturbations.

From a clinical perspective, spastic motor behaviors have traditionally been examined through single-joint 
movements, assuming they result from velocity-dependent stretch reflexes [24]. Results from the current study indicate that spastic reflexes also involve multisegmental interneuronal pathways, and thus, the management of spastic phenomena should include assessments of multijoint reflexes. More accurate assessments of spastic reflexes in SCI might be obtained through comprehensive evaluations that measure multiple reflex behaviors $[5,25]$.

\section{Involvement of Locomotor Pathways in the Reflexes Triggered by Hip and Knee Oscillation}

Hip proprioceptors have been shown to be a primary contributor to the reflex generation and modulation of muscle activity in spinalized animal models of locomotion. Alternating flexor and extensor activity is elicited by hip oscillation in spinalized and decerebrate cats [2627]. In particular, hip afferents are important for initiating the transition from stance to swing in spinalized cats during walking [28-30]. Further, the activation of flexor muscles during walking in decerebrate cats has been associated with afferent drive from the sartorius muscle [30-31]. Stretch of the sartorius muscle could contribute to the effects of hip/knee motion in the current study.

Recent studies in human SCI also indicate that multijoint reflexes triggered by unilateral hip oscillation entrain to the frequency of the imposed oscillation in a manner similar to spinalized animals [11-12]. The ability to modulate reflex activity in an organized pattern similar to locomotion suggests that reflex activity triggered by hip afferents may involve similar pathways for the spinal control of walking in human SCI [32]. In addition, hip kinematics modulate muscle activity in human SCI during treadmill stepping [33-34] such that swing is enhanced when hip extension is augmented during the late stance phase of gait $[33,35]$. In addition, while "hip walking" (knee joints fixed in an extended position), a muscle activity pattern similar to nondisabled walking is produced [33]; whether this would occur if the knee joints were fixed in a flexed position remains unclear. The muscle activity patterns produced by combined hip and knee extension movements observed in the current study are generally consistent with these previous reports.

Although not often emphasized, knee proprioceptors may also be involved in locomotor reflexes in human SCI. Imposed knee extension triggers a reflex response similar to imposed hip extension [4] or the combined hip and knee extension movement observed in the current study. Furthermore, the effects of modulation of phasing between the hip and knee joints on the reflex muscle activity suggest that both hip and knee afferents converge onto a common pathway at the spinal level. Specifically, the phasing between hip and knee affected the size of the spastic reflex muscle activity elicited by the hip and knee oscillation. The spastic reflex muscle activity pattern was more consistent with a locomotion pattern when the phasing between the hip and knee was close to the leg kinematics of nondisabled walking. These results suggest that the spastic reflexes triggered by the hip and knee oscillation may be partially involved in locomotor muscle activity and highlight the potential contribution of sensory information from hip and knee afferents to locomotor reflexes in human SCI. While this analogy with locomotion is potentially interesting, some inconsistencies existed between the current experimental setup and nondisabled walking conditions. For example, subjects were lying semireclined on a test bed and the ankle joint was secured in a stationary position with no loading input, which is quite different from walking.

Results from the current study are not completely consistent with the observations from animal studies. For instance, afferent information from the knee has not been shown to contribute significantly to the modulation of locomotor rhythms in the spinalized cat preparation [28]. However, activation of flexor muscles during walking in decerebrate cats has been associated with afferents drive from the sartorius muscle [30-31]. In addition, it remains unclear whether a muscle pattern similar to nondisabled walking would be produced by hip walking with knee joints locked in a flexed position in human SCI. On the basis of the results of the current study, we postulate that the magnitude of the muscle activity would be dramatically reduced.

The muscle activity produced by hip and knee oscillation, with the exception of the VM, was most appropriately phased for nondisabled walking when the phasing between hip and knee movement was closest to nondisabled walking. The muscle activity of the VM was antiphased with the hip position, even when the phasing between the hip and knee movement was similar to nondisabled walking. This dissimilarity of the reflex response with the gait pattern was also observed in the reflexes triggered by hip unilateral or bilateral oscillation in human SCI [11-12]. The activation of the VM during hip and knee extension might be associated with a permanent change in spinal reflex organization, since the VM activity also increases during a flexion withdrawal reflex [36]. 
Similarly, ankle load release, which produces a similar reflex response as hip stretch in animals models [37-38], also produces extensor spasms with increased VM activity in human SCI [18].

\section{Intersubject Variability}

Antispastic medications may have affected the magnitude of reflex responses for some subjects in the current study. At the time of the study, 4 of 11 subjects were prescribed baclofen to manage their spasms. Notably, one subject with an intrathecal baclofen pump was excluded from analysis because of the absence of extensor spasms following the hip and knee movement perturbations. In addition, level of injury has been suggested to affect locomotor EMG activity such that higher (cervical) lesion levels produce EMG activity more closely resembling nondisabled locomotor EMG patterns during treadmill stepping [39]. We observed no trends in the magnitude of the response with lesion level in the current study; however, our sample size may not have been sufficient to detect an effect of lesion location. No conclusions regarding antispastic medications, injury level, injury completeness (ASIA classification), or time postinjury could be made in the current study because of the high number of potential covariates, which limited the sample size for each subject type.

\section{Limitations}

Our conclusions are subject to several experimental limitations. For instance, although we validated the loadcell calibration using a standard weight and we have previously demonstrated the reliability of similar load-cell based techniques for measuring spastic reflexes [40], we did not systemically quantify the reliability of joint torque measurements with the apparatus used in the current study. Note that the hip torque measurements and the artifacts produced by the apparatus have previously been examined in detail [11-12]. The robotic arm moved the leg at a speed of $60^{\circ} / \mathrm{s}$ to elicit leg extensor spasms in the current study, although other faster speeds could also be used. The rationale for use of this speed was that spasms could be reliably elicited at $60 \%$, faster speeds produce larger inertial torque, and a previous study indicated that magnitude of extensor spasms in human SCI is not strongly velocity-dependent [4]. In addition, the repeated movements across all conditions might have impacted the magnitude of elicited spasms because of muscle fatigue or habituation of the response. To circumvent potential bias in the results from changes in the response over time, we randomized the order of the different experimental conditions, although we conducted the ramp and hold experiments first and the hip and knee oscillation experiments second.

\section{CONCLUSIONS}

Results from this study demonstrate that the position of the hip or knee modulates the spastic reflexes triggered by extension movement of the other joint (knee or hip), with larger responses observed with the opposite joint in the extended position. Such behaviors support the role of knee and hip proprioceptors, especially the biarticular sartorius muscle afferents, in the initiation of extensor spasms in human SCI. In addition, the reflex responses triggered by hip and knee oscillation are likely due to organized pathways located within the lumbosacral region of the spinal cord, possibly associated with locomotor reflexes.

\section{ACKNOWLEDGMENTS}

\section{Author Contributions:}

Study concept and design: M. Wu, B. Schmit.

Acquisition of data: M. Wu, B. Schmit.

Analysis and interpretation of data: M. Wu, B. Schmit.

Drafting of manuscript: $\mathrm{M}$. Wu.

Critical revision of manuscript for important intellectual content:

B. Schmit.

Statistical analysis: M. Wu.

Obtained funding: M. Wu, B. Schmit.

Administrative, technical, or material support: M. Wu, B. Schmit.

Study supervision: B. Schmit.

Financial Disclosures: The authors have declared that no competing interests exist.

Funding/Support: This material was based on work supported by the National Institute on Disability and Rehabilitation Research Switzer Distinguished Research Fellowship (grant H133F050031), Paralyzed Veterans of America Research Foundation (grant 2447), and the Falk Medical Research Trust.

Additional Contributions: We thank Ms. Tanya Onushko for help in the experimental setup.

Institutional Review: Informed consent was obtained and all procedures were conducted in accordance with the Helsinki Declaration of 1975 and approved by the institutional review boards of Northwestern University, Chicago, Illinois, and Marquette University, Milwaukee, Wisconsin.

Participant Follow-Up: The authors do not plan to inform participants of the publication of this study. However, participants have been encouraged to check the study Web site for updated publications. 


\section{REFERENCES}

1. Katz RT, Rymer WZ. Spastic hypertonia: Mechanisms and measurement. Arch Phys Med Rehabil. 1989;70(2):144-55. [PMID: 2644919]

2. Pandyan AD, Gregoric M, Barnes MP, Wood D, Van Wijck F, Burridge J, Hermens H, Johnson GR. Spasticity: Clinical perceptions, neurological realities and meaningful measurement. Disabil Rehabil. 2005;27(1-2):2-6. [PMID: 15799140] DOI:10.1080/09638280400014576

3. Schmit BD, Benz EN. Extensor reflexes in human spinal cord injury: Activation by hip proprioceptors. Exp Brain Res. 2002;145(4):520-27. [PMID: 12172664] DOI:10.1007/s00221-002-1134-5

4. Wu M, Hornby TG, Hilb J, Schmit BD. Extensor spasms triggered by imposed knee extension in chronic human spinal cord injury. Exp Brain Res. 2005;162(2):239-49. [PMID: 15586272] DOI:10.1007/s00221-004-2173-X

5. Benz EN, Hornby TG, Bode RK, Scheidt RA, Schmit BD. A physiologically based clinical measure for spastic reflexes in spinal cord injury. Arch Phys Med Rehabil. 2005;86(1):52-59. [PMID: 15640989]

DOI:10.1016/j.apmr.2004.01.033

6. Little JW, Micklesen P, Umlauf R, Britell C. Lower extremity manifestations of spasticity in chronic spinal injury. Am J Phys Med Rehabil. 1989;68(1):32-36. [PMID: 2917056] DOI:10.1097/00002060-198902000-00009

7. Barolat G, Maiman DJ. Spasms in spinal cord injury: A study of 72 subjects. J Am Paraplegia Soc. 1987;10(2):35-39. [PMID: 3450779]

8. Dimitrijevic MR, Lissens MA, Mckay WB. Characteristics and extent of motor activity recovery after spinal cord injury. In: Seil FJ, editor. Advances in neural regeneration research. New York (NY): Wiley-Liss; 1990. p. 381-405.

9. Kuhn RA. Functional capacity of the isolated human spinal cord. Brain. 1950;73(1):1-51. [PMID: 15420313] DOI:10.1093/brain/73.1.1

10. Sjölund BH. Pain and rehabilitation after spinal cord injury: The case of sensory spasticity? Brain Res Rev. 2002;40(1-3):250-56. [PMID: 12589923]

DOI:10.1016/S0165-0173(02)00207-2

11. Steldt RE, Schmit BD. Modulation of coordinated muscle activity during imposed sinusoidal hip movements in human spinal cord injury. J Neurophysiol. 2004;92(2):673-85.

[PMID: 15044520]

DOI:10.1152/jn.00677.2003

12. Onushko T, Schmit BD. Reflex response to imposed bilateral hip oscillations in human spinal cord injury. J Neurophysiol. 2007;98(4):1849-61. [PMID: 17652410] DOI:10.1152/jn.00461.2007
13. Batschelet E. Circular statistics in biology. New York (NY): Academic Press; 1981.

14. Frigo C, Pedotti A. Determination of muscle length during locomotion. In: Asmussen E, Jørjensen K, editors. Biomechanics VI. Baltimore (MD): University Park Press; 1978. p. $355-60$.

15. Hoy MG, Zajac FE, Gordon ME. A musculoskeletal model of the human lower extremity: The effect of muscle, tendon, and moment arm on the moment-angle relationship of musculotendon actuators at the hip, knee, and ankle. J Biomech. 1990;23(2):157-69. [PMID: 2312520] DOI:10.1016/0021-9290(90)90349-8

16. Perry J. Gait analysis: Normal and pathological function. Thorofare (NJ): SLACK; 1992.

17. Schmit BD, McKenna-Cole A, Rymer WZ. Flexor reflexes in chronic spinal cord injury triggered by imposed ankle rotation. Muscle Nerve. 2000;23(5):793-803. [PMID: 10797404] DOI:10.1002/(SICI)1097-4598(200005)23:5<793::AIDMUS18>3.0.CO;2-T

18. Wu M, Schmit BD. Spastic reflexes triggered by ankle load release in human spinal cord injury. J Neurophysiol. 2006; 96(6):2941-50. [PMID: 16855114]

DOI:10.1152/jn.00186.2006

19. Burke D, Gillies JD, Lance JW. The quadriceps stretch reflex in human spasticity. J Neurol Neurosurg Psychiatry. 1970;33(2):216-23. [PMID: 4245691]

DOI:10.1136/jnnp.33.2.216

20. Burke D, Gillies JD, Lance JW. Hamstrings stretch reflex in human spasticity. J Neurol Neurosurg Psychiatry. 1971; 34(3):231-35. [PMID: 4255176] DOI:10.1136/jnnp.34.3.231

21. Schmit BD, Benz EN, Rymer WZ. Afferent mechanisms for the reflex response to imposed ankle movement in chronic spinal cord injury. Exp Brain Res. 2002;145(1):40-49. [PMID: 12070743] DOI:10.1007/s00221-002-1080-2

22. Wu M, Hornby TG, Kahn JH, Schmit BD. Flexor reflex responses triggered by imposed knee extension in chronic human spinal cord injury. Exp Brain Res. 2006;168(4): 566-76. [PMID: 16151779] DOI:10.1007/s00221-005-0113-z

23. Sköld C, Levi R, Seiger A. Spasticity after traumatic spinal cord injury: Nature, severity, and location. Arch Phys Med Rehabil. 1999;80(12):1548-57. [PMID: 10597805] DOI:10.1016/S0003-9993(99)90329-5

24. Lance JW. Pathophysiology of spasticity and clinical experience with baclofen. In: Feldman RG, Young RR, Koella WP, editors. Spasticity: Disordered motor control. Chicago (IL): Year Book Medical Publishers; 1980. p.185-204.

25. Priebe MM, Sherwood AM, Thornby JI, Kharas NF, Markowski J. Clinical assessment of spasticity in spinal cord injury: A multidimensional problem. Arch Phys Med 
Rehabil. 1996;77(7):713-16. [PMID: 8670001]

DOI:10.1016/S0003-9993(96)90014-3

26. Andersson O, Grillner S. Peripheral control of the cat's step cycle. II. Entrainment of the central pattern generators for locomotion by sinusoidal hip movements during "fictive locomotion.” Acta Physiol Scand. 1983;118(3):229-39. [PMID: 6312752] DOI:10.1111/j.1748-1716.1983.tb07267.x

27. Kriellaars DJ, Brownstone RM, Noga BR, Jordan LM. Mechanical entrainment of fictive locomotion in the decerebrate cat. J Neurophysiol. 1994;71(6):2074-86. [PMID: 7931503]

28. Grillner S, Rossignol S. On the initiation of the swing phase of locomotion in chronic spinal cats. Brain Res. 1978; 146(2):269-77. [PMID: 274169] DOI:10.1016/0006-8993(78)90973-3

29. Hiebert GW, Whelan PJ, Prochazka A, Pearson KG. Contribution of hind limb flexor muscle afferents to the timing of phase transitions in the cat step cycle. J Neurophysiol. 1996;75(3):1126-37. [PMID: 8867123]

30. Lam T, Pearson KG. Proprioceptive modulation of hip flexor activity during the swing phase of locomotion in decerebrate cats. J Neurophysiol. 2001;86(3):1321-32. [PMID: 11535680]

31. Lam T, Pearson KG. Sartorius muscle afferents influence the amplitude and timing of flexor activity in walking decerebrate cats. Exp Brain Res. 2002;147(2):175-85. [PMID: 12410332] DOI:10.1007/s00221-002-1236-0

32. Knikou M, Kay E, Schmit BD. Parallel facilitatory reflex pathways from the foot and hip to flexors and extensors in the injured human spinal cord. Exp Neurol. 2007;206(1): 146-58. [PMID: 17543951] DOI:10.1016/j.expneurol.2007.05.004

33. Dietz V, Müller R, Colombo G. Locomotor activity in spinal man: Significance of afferent input from joint and load recep- tors. Brain. 2002;125(Pt 12):2626-34. [PMID: 12429590] DOI:10.1093/brain/awf273

34. Harkema SJ, Hurley SL, Patel UK, Requejo PS, Dobkin $\mathrm{BH}$, Edgerton VR. Human lumbosacral spinal cord interprets loading during stepping. J Neurophysiol. 1997;77(2): 797-811. [PMID: 9065851]

35. Dobkin BH, Harkema S, Requejo P, Edgerton VR. Modulation of locomotor-like EMG activity in subjects with complete and incomplete spinal cord injury. J Neurol Rehabil. 1995;9(4):183-90. [PMID: 11539274]

36. Deutsch KM, Hornby TG, Schmit BD. The intralimb coordination of the flexor reflex response is altered in chronic human spinal cord injury. Neurosci Lett. 2005;380(3):305-10.

[PMID: 15862907] DOI:10.1016/j.neulet.2005.01.060

37. Conway BA, Hultborn H, Kiehn O. Proprioceptive input resets central locomotor rhythm in the spinal cat. Exp Brain Res. 1987;68(3):643-56. [PMID: 3691733] DOI:10.1007/BF00249807

38. Duysens J, Pearson KG. Inhibition of flexor burst generation by loading ankle extensor muscles in walking cats. Brain Res. 1980;187(2):321-32. [PMID: 7370733] DOI:10.1016/0006-8993(80)90206-1

39. Dietz V, Nakazawa K, Wirz M, Erni T. Level of spinal cord lesion determines locomotor activity in spinal man. Exp Brain Res. 1999;128(3):405-9. [PMID: 10501813] DOI:10.1007/s002210050861

40. Starsky AJ, Sangani SG, McGuire JR, Logan B, Schmit BD. Reliability of biomechanical spasticity measurements at the elbow of people poststroke. Arch Phys Med Rehabil. 2005;86(8):1648-54. [PMID: 16084821] DOI:10.1016/j.apmr.2005.03.015

Submitted for publication July 8, 2009. Accepted in revised form December 8, 2009. 
